\title{
Arbitrary Power Splitting Couplers Based on 3x3 Multimode Interference Structures for All-optical Computing
}

\author{
Trung-Thanh Le
}

\begin{abstract}
Chip level optical links based on VLSI photonic integrated circuits have been proposed to replace metal electrical data paths in cases where high frequencies make electrical traces impractical. This is especially relevant to highspeed clock signals as silicon CMOS circuit technology is scaled higher in speed to the $\mathrm{GHz}$ range and beyond. In this paper, the realization of optical couplers and power taps based on 3x3 multimode interference structures using CMOS technology is presented. The proposed devices can be useful for all-optical interconnects, clock distribution and many other all-optical processing applications. The transfer matrix method and the 3D Beam Propagation Method (3D BPM) are used to optimize the proposed devices.
\end{abstract}

Index Terms--All-optical processing, CMOS technology, Integrated optics, multimode interference (MMI) couplers

\section{INTRODUCTION}

The recent emergence of chip multiprocessors that obtain a better performance increasing the number of computational cores has changed the trend in system interconnects and global communications infrastructure. Chip multiprocessor architectures reach high parallel computing and their performance is directly tied to how efficiently the parallelism of the system is exploited and its aggregate compute power used. The realization of a scalable on-chip and off-chip communication infrastructure faces critical challenges in meeting the enormous bandwidths, capacities, and stringent latency requirements demanded by chip multiprocessors maintaining a suitable performanceper-watt. The importance of improving a low-power communication infrastructure for those next generation multiprocessors lets photonic Networks on Chip using complementary metal-oxide-semiconductor technology offer a promising solution.

Current integrated photonic technology presents huge advancements in fabrication capabilities of nano-scale devices and precise control over their optical properties. Importantly, these breakthroughs have led to the development of silicon photonic device integration with electronics directly in commercial complementary metaloxide-semiconductor technology. Silicon photonics [1-3] was chosen because the fabrication of such devices require only small and low cost modifications to existing fabrication processes. SOI technology is compatible with existing complementary metal-oxide-semiconductor technologies for making compact, highly integrated, and multifunction devices $[4,5]$. The SOI platform uses silicon both as the substrate and the guiding core material. The large index

Manuscript received August 7, 2011, revised September 9, 2011.

Trung-Thanh Le is with the Faculty of Information Technology, Hano University of Natural Resources and Environment, 41A K1 Phu Dien Road, Tu Liem, Ha Noi, Viet Nam (Email: thanh.le@hunre.edu.vn) contrast between $\mathrm{Si}\left(\mathrm{n}_{\mathrm{Si}}=3.45\right.$ at wavelength $\left.1550 \mathrm{~nm}\right)$ and $\mathrm{SiO}_{2}\left(\mathrm{n}_{\mathrm{SiO}_{2}}=1.46\right)$ allows light to be confined within submicron dimensions and single mode waveguides can have core cross-sections with dimensions of only few hundred nanometres and bend radii of a few micrometers with minimal losses. Moreover, silicon nano-photonics offer potential for monolithic integration of electronic and photonic devices on a single substrate.

The multimode interference effect based devices have the advantages of low lost, compactness and cost-effective solutions. The realization of optical couplers and power taps based on $2 \times 2$ multimode interference (MMI) structures has been given in the literature [6]. However, these proposed devices required to have long pattern length and large size.

In this paper, optical coupler and power taps with arbitrary power splitting ratios based on $3 \times 3$ multimode interference (MMI) structures using CMOS technology are proposed. The variable coupling feature of the optical tap makes it ideal for high speed clock distribution, whereby an optical clock signal is distributed via optical paths to multiple tap points on an electronic circuit board or chip. The transfer matrix method and the 3D-BPM are used to design and optimize the device.

\section{THEORY}

The MMI coupler consists of a multimode optical waveguide that can support a number of modes. In order to launch and extract light from the multimode region, a number of single mode access waveguides are placed at the input and output planes. If there are $\mathrm{N}$ input waveguides and $\mathrm{M}$ output waveguides, then the device is called an $\mathrm{NxM}$ MMI coupler.

The layout of a 2x2 MMI coupler with arbitrary coupling ratios based on $3 \times 3$ MMI couplers is shown in Fig. 1. This MZI structure consists of three sections: a 3x3 MMI splitter, a phase shifting region with two phase shifters and a $3 \times 3$ MMI combiner. Here $a_{i}(i=1,2,3)$ and $b_{j}(j=1,2,3)$ are the complex amplitudes of the signals at input and output ports, respectively.

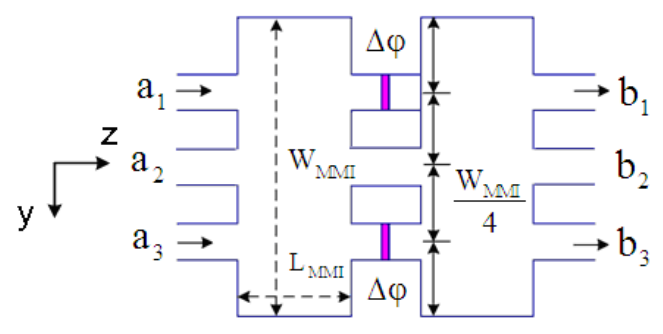

Fig. 1. A 2x2 MMI coupler based on 3x3 RI-MMI couplers

The power at the output ports can be controlled simply by adjusting the phase shifters in the outer linking waveguides 
or in the central linking waveguide. The phase $\phi_{\mathrm{ij}}$ and amplitudes $\mathrm{A}_{\mathrm{ij}}$ associated with imaging from input $\mathrm{i}$ to output $\mathrm{j}$ in an MMI coupler having a length of $\mathrm{L}_{\mathrm{MMI}}=3 \mathrm{~L}_{\pi} / 8$ have been given by Bachmann et al. [7].

The phases $\phi_{\mathrm{ij}}$ and the respective amplitudes $\mathrm{A}_{\mathrm{ij}}$ form a transfer matrix $\mathbf{M}$ describing the characteristic of the MMI coupler.

The complex optical field amplitudes at the output ports are then related to the complex optical field amplitudes at the input ports in the structure in Fig. 1 by

$$
\mathbf{b}=\mathbf{S a}
$$

where $\mathbf{b}=\left[\begin{array}{lll}b_{1} & b_{2} & b_{3}\end{array}\right]^{T}, \quad \mathbf{a}=\left[\begin{array}{lll}a_{1} & a_{2} & a_{3}\end{array}\right]^{T}$ and $\mathbf{S}=\mathbf{M \Phi M ~ . ~}$ Here, $\mathbf{M}$ and $\boldsymbol{\Phi}$ are the transfer matrices for the MMI couplers and phase shift elements, respectively. The transfer matrix $\mathbf{M}$ can be written as

$$
\mathbf{M}=\left[\begin{array}{ccc}
\frac{j}{2} & \frac{j}{\sqrt{2}} e^{-j \frac{3 \pi}{8}} & -\frac{j}{2} \\
\frac{j}{\sqrt{2}} e^{-j \frac{3 \pi}{8}} & 0 & \frac{j}{\sqrt{2}} e^{-j \frac{3 \pi}{8}} \\
-\frac{j}{2} & \frac{j}{\sqrt{2}} e^{-j \frac{3 \pi}{8}} & \frac{j}{2}
\end{array}\right]
$$

The phase shifters may be located in the two outer arms or alternatively, in the middle (or central) arm. For example, the phases of the signals propagating through the outer arms may be shifted by an amount of $\Delta \varphi$. The action of these phase shifters can described by a matrix $\boldsymbol{\Phi}$

$$
\boldsymbol{\Phi}=\left[\begin{array}{ccc}
\mathrm{e}^{\mathrm{j} \Delta \varphi} & 0 & 0 \\
0 & 1 & 0 \\
0 & 0 & \mathrm{e}^{\mathrm{j} \Delta \varphi}
\end{array}\right]
$$

It is clear from equation (1) that a $2 \times 2$ MMI coupler is achieved if the outer access waveguides (port 1 and port 3 ) are used. The overall transfer matrix of this $2 \times 2$ MMI coupler then may be written as

$$
\mathbf{S}=\left[\begin{array}{cc}
-0.5 \mathrm{e}^{\mathrm{j} \Delta \varphi}-0.5 \mathrm{e}^{-\mathrm{j} \frac{3 \pi}{4}} & 0.5 \mathrm{e}^{\mathrm{j} \Delta \varphi}-0.5 \mathrm{e}^{-\mathrm{j} \frac{3 \pi}{4}} \\
0.5 \mathrm{e}^{\mathrm{j} \Delta \varphi}-0.5 \mathrm{e}^{-\mathrm{j} \frac{3 \pi}{4}} & 0.5 \mathrm{e}^{\mathrm{j} \Delta \varphi}+0.5 \mathrm{e}^{-\mathrm{j} \frac{3 \pi}{4}}
\end{array}\right]
$$

The relations between the complex input and output amplitudes are given by

$$
\left[\begin{array}{l}
b_{1} \\
b_{3}
\end{array}\right]=\mathbf{S}\left[\begin{array}{l}
a_{1} \\
a_{2}
\end{array}\right]
$$

For an input signal presented at input port 1, the normalized output powers at the bar and cross ports are $\mathrm{P}_{\mathrm{b}}=\left|\mathrm{b}_{1}\right|^{2}$ and $\mathrm{P}_{\mathrm{c}}=\left|\mathrm{b}_{2}\right|^{2}$. Theoretically, these powers will vary with the phase shift $\Delta \varphi$ as shown in Fig. 2. It is clear from this figure that the output powers can be controlled theoretically over a full range from 0 to 1 by adjusting the phase shift value $\Delta \varphi$ from $-3 \pi / 4$ to $\pi / 4$. This is a general concept which provides the basis for the later design of MMI couplers with arbitrary coupling ratios.

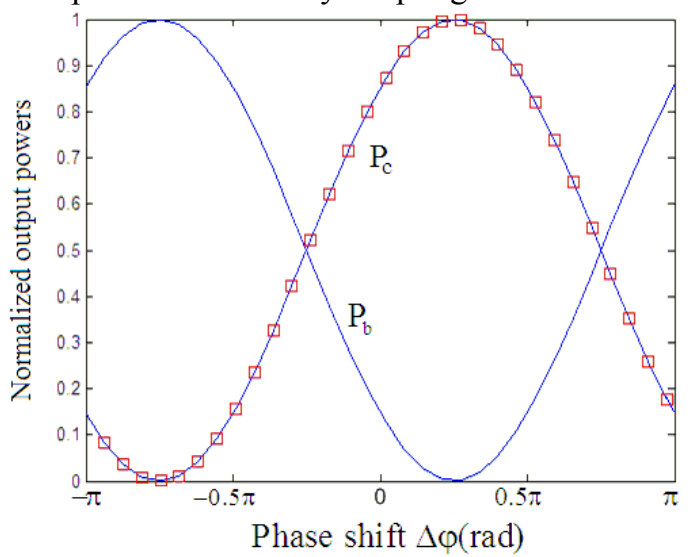

Fig. 2. The dependence of the output powers on the phase shifts

\section{SimULATION RESUltS}

It is well known that the finite-difference time-domain (FDTD) method is a general method to solve Maxwell's partial differential equations numerically in the time domain. Simulation results for devices on the SOI channel waveguide using the 3D-FDTD method can achieve a very high accuracy. However, due to the limitation of computer resources and memory requirements, it is difficult to apply the 3D-FDTD method to the modelling of large devices on the SOI channel waveguide. Meanwhile, the 3D-BPM was shown to be a quite suitable method that has sufficient accuracy for simulating devices based on SOI channel waveguides $[8,9]$. Therefore, the design for devices on the SOI platform will now be performed using the 3D-BPM. The theory of the 3D-BPM is studied in detail in [10]. However, in this study, a different implementation algorithm for the 3D-BPM will be used.

The waveguide structure used in the designs is shown in Fig. 3. Here, $\mathrm{SiO}_{2}\left(\mathrm{n}_{\mathrm{SiO}_{2}}=1.46\right)$ is used as the upper cladding material. An upper cladding region is used to avoid the influence of moisture and environmental temperature. The parameters used in the designs are as follows: the waveguide has a standard silicon thickness of $h_{\text {co }}=220 \mathrm{~nm}$ and access waveguide widths are $\mathrm{W}_{\mathrm{a}}=0.48 \mu \mathrm{m}$ for single mode operation. It is assumed that the designs are for the transverse electric (TE) polarization at a central optical wavelength $\lambda=1550 \mathrm{~nm}$.

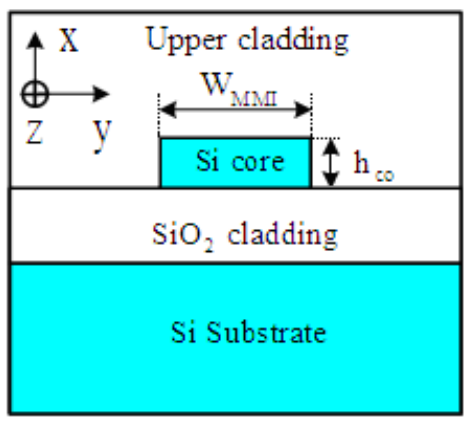

Fig. 3. Silicon waveguide cross-section used in the designs of the proposed device 
By forming a surface pattern on the top of the MMI region at special positions or on the top of linking waveguides (SOI channel waveguides), any desired phase shift can be produced [11]. The refractive index is adjusted by changing the etch depth, etch width and/or the length of these patterns. The advantages of this approach are that the etching can be done after the device has been fabricated and patterns introduce only low additional loss. In addition, by using suitable masks, only one additional simple process step is required.

By using the 3D-BPM simulation, the effective refractive indices for the TE fundamental mode for different waveguide thicknesses, but the same width of $480 \mathrm{~nm}$, are plotted in Fig. 4. The simulations show that the effective refractive index is directly proportional to the waveguide thickness. This is the operating principle of introducing a phase shift using the surface pattern technique.

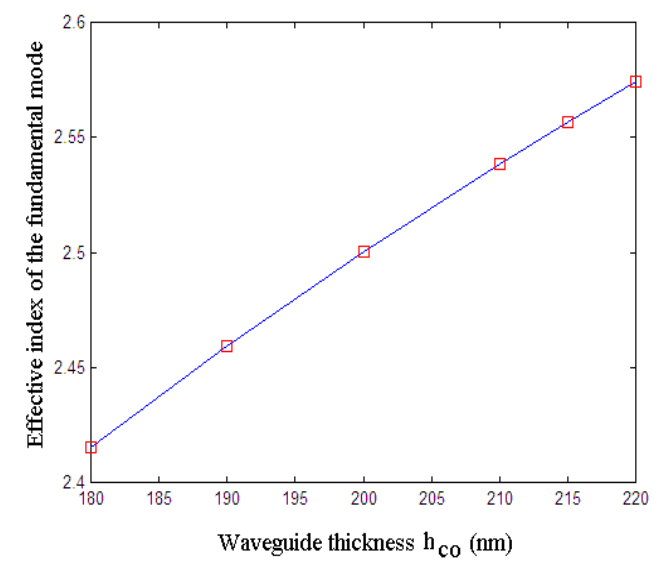

Fig. 4. The effective indices of the fundamental TE mode at different waveguide thicknesses for an SOI channel waveguide of width $480 \mathrm{~nm}$

In order to achieve a phase shift of $\Delta \varphi=-\pi$, the length $L_{p}$ of the pattern region as a function of the pattern depths $h_{p}$ $\left(\mathrm{h}_{\mathrm{p}}=\mathrm{h}_{\mathrm{co}}-\mathrm{h}_{\mathrm{pt}}\right)$ calculated from $\mathrm{L}_{\mathrm{p}}=\frac{\lambda}{2 \pi} \frac{\Delta \varphi}{\Delta \mathrm{n}_{\mathrm{e}}}$ is shown in Fig. 5. Here, $\Delta \mathrm{n}_{\mathrm{e}}$ is the difference in effective indices between that for the waveguide having a thickness $h_{\text {co }}$ and that for the standard waveguide having a thickness of $\mathrm{h}_{\mathrm{co}}=220 \mathrm{~nm}$.

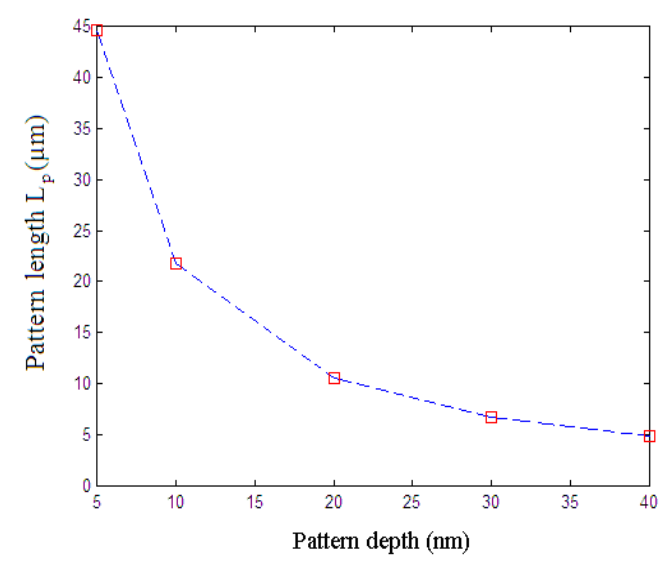

Fig. 5. Pattern length $L_{p}$ required to achieve a phase shift of - $\pi$ at different depths

The 3D-BPM simulation result (Fig. 6) shows that at a pattern length of $\mathrm{L}_{\mathrm{p}}=10 \mu \mathrm{m}$, the loss of the signal propagating through the pattern section is only $0.043 \mathrm{~dB}$. A pattern depth of $h_{p}=40 \mathrm{~nm}$ is used throughout this chapter.

These dimensions are suitable for practical fabrication.

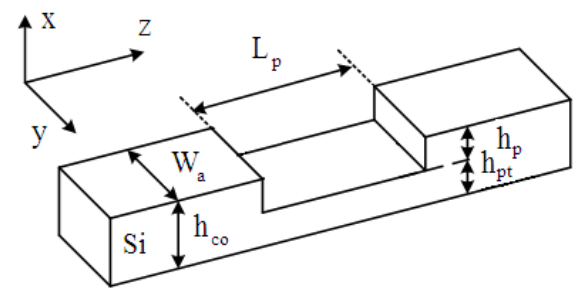

(a) Structure of the pattern region

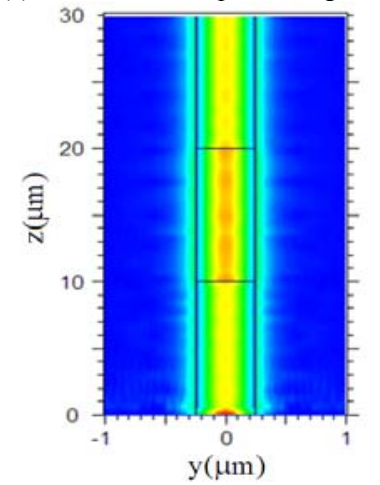

(b) Field distribution through the pattern

Fig. 6. Pattern used for introducing a phase shift (a) structure of the pattern region and (b) power distribution through a pattern having a length of

$$
\mathrm{L}_{\mathrm{p}}=10 \mu \mathrm{m}
$$

In order to produce a phase shift $\Delta \varphi$ varying from $-\frac{3 \pi}{4}$ to 0 , the patterns must be made in the outer linking arms of the MZI structure in Fig. 6. To produce a phase shift $\Delta \varphi$ varying from 0 to $\frac{\pi}{4}$, then the pattern must be made in the centre arm. If the phase shifts are to be achieved using the surface pattern technique on the linking waveguides, then the normalized output powers can be plotted as functions of pattern length $\mathrm{L}_{\mathrm{p}}$ as shown in Fig. 7. The results show that the full range of power coupling ratios $|\kappa|^{2}$ should be achievable with an accumulated pattern length of less than $5 \mu \mathrm{m}$.

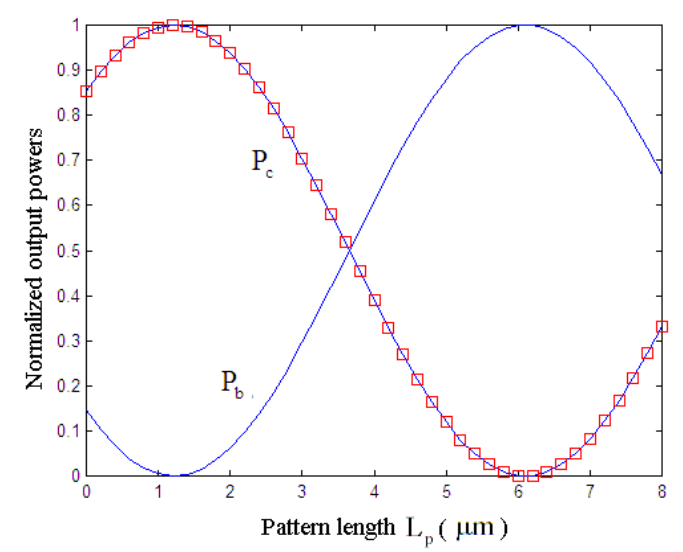

Fig. 7. Output powers as functions of the pattern length $L_{p}$ 
The design procedures for the access waveguides, MMI sections and the tapered waveguides are similar to those of the previous section. The pattern regions can be implemented on the top of the waveguide arms or within an MMI region.

The following two examples show how the theory developed so far can be applied to designing couplers with arbitrary coupling ratios. The design process commences with the optimisation of the design of individual $3 \times 3 \mathrm{MMI}$ couplers. The MMI width is chosen to be $\mathrm{W}_{\mathrm{MMI}}=4 \mu \mathrm{m}$. By using the MPA method, the length of the $3 \times 3$ MMI coupler is determined first. The MMI length calculated by using the MPA method [12] is $\mathrm{L}_{\mathrm{MPA}}=3 \mathrm{~L}_{\pi} / 8=15.3 \mu \mathrm{m}$. Then the 3D-BPM is used to simulate the device where the MMI length is varied around $3 \mathrm{~L}_{\pi} / 8$. The aim is to optimise the length of the MMI structures to achieve the best performance possible. The optimised length of the two $3 \times 3$ MMI couplers is found to be $\mathrm{L}_{\mathrm{MMI}}=14.67 \mu \mathrm{m}$. These couplers are then used in the MZI structure. In the first example, the 3D-BPM simulation for the device having no pattern region is shown in Fig. 8. The 3D-BPM simulation shows that the output powers at the bar and cross ports are 0.11 and 0.81 , respectively. The computed excess loss is $0.36 \mathrm{~dB}$.

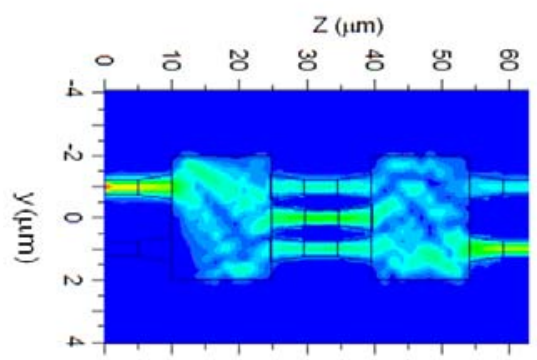

Fig. 8. 3D-BPM simulation of a $2 \times 2$ MMI coupler based on a $3 \times 3 \mathrm{MMI}-$ MZI structure with no pattern region made in the linking waveguides of the device

In the second case, a pattern having a length of $\mathrm{L}_{\mathrm{p}}=4 \mu \mathrm{m}$ is made in the middle (central) arm of the MZI structure. The phase shift $\Delta \varphi$ as calculated from the above theory is close to $-3 \pi / 4$. This means that the power is completely transferred to the bar port. The 3D-BPM simulation result for this case is plotted in Fig. 9. The computed excess loss is about $0.4 \mathrm{~dB}$.

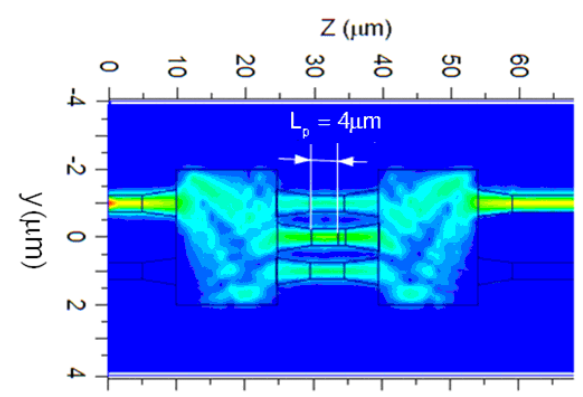

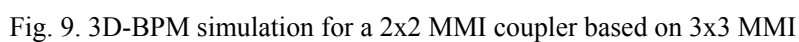
couplers having a pattern length of $\mathrm{L}_{\mathrm{p}}=4 \mu \mathrm{m}$ made at the middle linking arm

An alternative to patterning the linking waveguides is to join the two MMI sections and make the patterns within the
MMI section, as shown in Fig. 10. The required pattern positions within the MMI can be found from this 3D-BPM simulation.

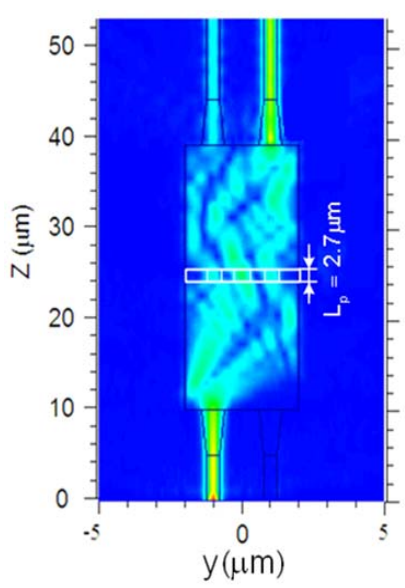

Fig. 10. 3D-BPM calculation for the pattern positions

The power distribution in Fig. 10 shows that the maximum useful length of the pattern made directly within the MMI region is around $L_{p}=2.7 \mu \mathrm{m}$. This results in the possibility of varying the power coupling ratio over a range from 0.15 to 0.85 as shown in Fig. 7. The 3D-BPM is then used to investigate the device performance in the case of zero pattern length $\mathrm{L}_{\mathrm{p}}=0 \mu \mathrm{m}$. The 3D-BPM simulation (Fig. 10) shows that the computed normalized output powers are 0.12 for the bar port and 0.82 for the cross port, respectively. The computed excess loss is $0.27 \mathrm{~dB}$ in this case.

\section{CONCLUSION}

This paper has proposed a novel method for realizing optical power couplers and taps with arbitrary power splitting ratios based on $3 \times 3$ multimode interference using CMOS technology. The design of the proposed devices has been verified and optimised using numerical simulation methods. These devices can be useful for all-optical computing systems, clock distribution and VLSI photonic integrated circuits.

\section{REFERENCES}

[1] L. Pavesi and D. J. Lockwood, Silicon Photonics: Springer, 2004.

[2] G. T. Reed and A. P. Knights, Silicon Photonics: An Introduction: John Wiley and Sons, March 2004.

[3] T. Tsuchizawa, K. Yamada, and H. F. e. al., "Microphotonics Devices Based on Silicon Micro-Fabrication Technology," IEEE Journal of Selected Topics in Quantum Electrononics, vol. 11, pp. 232-, 2005.

[4] S. Janz, P. Cheben, and D. D. e. al., "Microphotonic elements for integration on the silicon-on-insulator waveguide platform," IEEE Journal of Selected Topics in Quantum Electrononics, vol. 12, pp. 1402-1415, Dec. 2006.

[5] R. Soref, "The past, present, and future of silicon photonics," IEEE Journal of Selected Topics in Quantum Electrononics, vol. 12, pp. 1678-1687, Dec. 2006.

[6] T. T. Le, L. W. Cahill, and D. Elton, "The Design of 2x2 SOI MMI couplers with arbitrary power coupling ratios," Electronics Letters, vol. 45, pp. 1118-1119, 2009.

[7] M. Bachmann, P. A. Besse, and H. Melchior, "Overlapping-image multimode interference couplers with a reduced number of selfimages for uniform and nonuniform power splitting," Applied Optics, vol. 34, pp. 6898-6910, 1995.

[8] E. Dulkeith, F. Xia, and L. S. e. al., "Group index and group velocity dispersion in silicon-on-insulator photonic wires," Optics Express, vol. 14, pp. 3853-3863, 2006. 
[9] J. I. Dadap, N. C. Panoiu, and X. C. e. al., "Nonlinear-optical phase modification in dispersion-engineered $\mathrm{Si}$ photonic wires," Optics Express, vol. 16, pp. 1280-1299, 2008.

[10] W. P. Huang, C. L. Xu, and S. K. Chaudhuri, "A finite-difference vector beam propagation method for three-dimensional waveguide structures," IEEE Photonics Technology Letters, vol. 4, pp. 148 - 151, 1992.

[11] T. T. Le and L. W. Cahill, "The Design of Multimode Interference Couplers with Arbitrary Power Splitting Ratios on an SOI Platform," presented at LEOS 2008, Newport Beach, California, USA, 9-14 Nov 2008.

[12] L. W. Cahill and T. T. Le, "Modal Propagation Analysis Method for the Design of MMI Coupler Based Microring Resonators," presented at Progress In Electromagnetics Research Symposium (PIERS) Proceedings, Cambridge, USA, July 2-6, 2008.

Trung-Thanh Le received the B.Sc. and M.Sc. degrees in electronic and telecommunication engineering from Hanoi University of Technology, Vietnam in 2003 and 2005, respectively. He received the $\mathrm{PhD}$ degree in electronic engineering from La Trobe University, Australia in 2009. He was a lecturer at the University of Transports and Communications, Hanoi, Vietnam from 2003-2010 and since 2010, he is the Dean in charge of the Faculty of Information Technology, Hanoi University of Natural Resources and Environment, Hanoi, Vietnam. 